\title{
MORAL VALUES.*
}

BY R. HENDERSON.

THE question of moral values in connection with the Theory of Probability is one which has given rise to great diversity of opinion among mathematicians. In Bertrand's Calcul des Probabilités, which is, perhaps, the most important work of recent years on the subject of Probability, it is dismissed with contempt, while in some other works considerable space is devoted to it.

Perhaps one of the reasons for the disrepute in which some hold the theory of moral values, is the fact that almost all who have taken up the subject from a mathematical standpoint have adhered to Bernoulli's or some other equally rigid hypothesis. Bernoulli's hypothesis is that the increase in moral value, arising from an indefinitely small increase in wealth, varies directly as that increase and inversely as the wealth already in possession, or, in other words, denoting the moral value of a wealth $x$ by the symbol $f(x)$, that $\frac{d f(x)}{d x}=\frac{a}{x}$, where $a$ is a constant, or, integrating this, that $f(x)=\alpha \log x+c, c$ being another arbitrary constant. The form of $f(x)$ adopted by Cramer was $f(x)=a x^{\frac{1}{2}}$. It would seem that many, being unwilling to admit these hypotheses, have been inclined to ridicule the whole subject, and have been to a certain extent blind to the basis of fact on which it is founded.

Now it has long seemed to me that Bernoulli did not intend his hypothesis as an absolute statement of the law of moral values, but rather as merely a particular case of a more general hypothesis, however definite or indefinite in his mind that general hypothesis may have been. And indeed writers on the subject of Economics, to whose department the question of the admissibility, or the contrary, of the hypothesis properly belongs, have adopted, though I cannot say they have originated, a more general hypothesis treated of below.

The earliest statement of this general hypothesis which I have seen is in the introduction to Laplace's Théorie Analytique des Probabilités, page xxii of the edition of 1847, at which place there occurs a passage of which the following is a free translation:

"The disadvantage of gambling and of exposing to the same risk all goods to be shipped, and all similar results indicated by good sense, subsist, whatever function the moral value may be of the material fortune of each individual. It is sufficient that the ratio of the increase of that function to

* Presented to the American Mathematical Society, October 26, 1895. 
the increase of the material fortune diminish as this latter increases."

Professor Marshall in his Principles of Economics, Appendix, Note ix, by a course of reasoning similar to that below, proves the disadvantage of gambling, provided $f(x)$ is amenable to expansion in Taylor's series in the form which he uses, that is, provided $\frac{d^{2} f(x)}{d x^{2}}$ is continuous and finite; but I have not been able to find anywhere a complete proof of the truth of these statements of Laplace, and it might be interesting to examine them analytically.

Denoting, as above, the moral value of a material fortune of amount $x$, by $f(x)$, Laplace's statement is equivalent to saying that it is sufficient that $\frac{d f(x)}{d x}$ should diminish as $x$ increases, or, in other words, that $\frac{d^{2} f(x)}{d x^{2}}$ should be always negative, but not necessarily finite nor continuous. I shall introduce the extra assumption that $\frac{d f(x)}{d x}$ is always finite which enables me to use the following expression for $f(x+h)$, the source of which I cannot at this moment recollect.

$$
\begin{aligned}
f(x+h) & =f(x)+\int_{0}^{h} f^{\prime}(x+h-z) d z \\
& =f(x)+h f^{\prime}(x)+\int_{0}^{h} z f^{\prime \prime}(x+h-z) d z \\
& =f(x)+h f^{\prime}(x)+\theta h \int_{0}^{h} f^{\prime \prime}(x+h-z) d z \\
& =f(x)+h f^{\prime}(x)+\theta h\left\{f^{\prime}(x+h)-f^{\prime}(x)\right\},
\end{aligned}
$$

where $\theta$ is a proper fraction, since $f^{\prime \prime}(x+h-z)$ retains the same sign throughout. And since that sign is negative, the sign of $\int_{0}^{h} f^{\prime \prime}(x+h-z) d z$ is necessarily different from that of $h$, and consequently the last term $\theta h\left\{f^{\prime}(x+h)-f^{\prime}(x)\right\}$ is essentially negative. These results hold subject to the condition above stated that $f^{\prime}(x)$ should be always finite.

This equation then may be expressed in the form

$$
f(x+h)<f(x)+h f^{\prime}(x)
$$

for all values of $h$. From this inequality it is easy to deduce the following theorem:

Theorem. If $f(x)$ be a function of $x$ such that $f^{\prime}(x)$ is finite and $f^{\prime \prime}(x)$ is negative for all values of $x$, then for all positive values of $l$ and $m$ we have

$$
\frac{l f(\alpha)+m f(b)}{l+m}<f\left(\frac{l a+m b}{l+m}\right)
$$


For, let $\frac{l a+m b}{l+m}=x$ and $\frac{b-a}{l+m}=h$,

then

$$
a=x-m h \text { and } b=x+l h,
$$

$$
\text { and } \begin{aligned}
\frac{l f(a)+m f(b)}{l+m} & =\frac{l}{l+m} f(x-m h)+\frac{m}{l+m} f(x+l h) \\
& <\frac{l}{l+m}\left\{f(x)-m h f^{\prime}(x)\right\}+\frac{m}{l+m}\left\{f(x)+l h f^{\prime}(x)\right\},
\end{aligned}
$$

i.e. $<f(x)$,

i.e. $<f\left(\frac{l a+m b}{l+m}\right)$.

Corollary. Applying this theorem successively, we get

$$
\frac{l f(a)+m f(b)+n f(c)+\cdots}{l+m+n+\cdots}<f\left(\frac{l a+m b+n c+\cdots}{l+m+n+\cdots}\right),
$$

where $l, m, n$, etc., are all positive.

Supposing now that the moral value of a material fortune $x$ may be represented by $f(x)$, a function which satisfies the above conditions, we have the following results :

(1) $A$ bet at fair odds is morally disadvantageous.

For, let the man's material fortune before making the bet be $a$, and let $x$ be staked against $y$ in a fair wager.

Then the probability of winning is $\frac{x}{x+y}$, and of losing is $\frac{y}{x+y}$. Assuming then that the moral value of his expectation is equal to the sum of the moral values of the various amounts of which he may ultimately stand possessed multiplied respectively by their probabilities, we have it equal, after the bet is made, to

$$
\frac{x}{x+y} f(a+y)+\frac{y}{x+y} f(a-x),
$$

which by the theorem is less than

$$
f\left(\frac{x}{x+y}(a+y)+\frac{y}{x+y}(a-x)\right)
$$

or $f(\alpha)$ the moral value at first.

(2) If a merchant expects a shipment, the moral value of his expectation is greater if the shipment be divided than if it be shipped in one vessel only, the probability of each vessel arriving being the same. 
Let $v$ be the merchant's wealth in possession, $s$ the value of the shipment, and $p$ the probability of each vessel arriving, and let $(1-p)=q$.

Then if it be shipped in one vessel, the moral value of his expectation is $p f(v+s)+q f(v)$.

But if it be shipped in $t$ different vessels,

The probability of

all arriving is $p^{t}$, when his wealth will be $(v+s)$.

$$
\begin{aligned}
& t-1 \quad \text { " } t p^{t-1} q, \quad \text { " } \quad\left(v+\frac{t-1}{t} s\right) \text {. } \\
& t-2 \quad \text { " } \frac{t(t-1)}{1 \cdot 2} p^{t-2} q^{2}, \quad \text { " } \quad\left(v+\frac{t-2}{t} s\right) \text {. }
\end{aligned}
$$

etc.

Therefore the moral value of his expectation is

$$
\begin{aligned}
& p^{t} f(v+s)+t p^{t-1} q f\left(v+\frac{t-1}{t} s\right)+\frac{t(t-1)}{1 \cdot 2} f\left(v+\frac{t-2}{t} s\right) \\
&+\cdots+q^{t} f(v), \\
&>p^{t} f(v+s)+\left\{(t-1) p^{t-1} q f(v+s)+p^{t-1} q f(v)\right\} \\
&+\left\{\frac{(t-1)(t-2)}{1 \cdot 2} f(v+s)+\frac{t-1}{1} f(v)\right\}+\cdots+q^{t} f(v), \\
& \text { i.e. }>p(p+q)^{t-1} f(v+s)+q(p+q)^{t-1} f(v), \\
& \text { i.e. }>p f(v+s)+q f(v),
\end{aligned}
$$

which is the moral value of his expectation if the shipment be in one vessel only.

(3) In the above case, the larger the number of vessels, the greater is the moral value of his expectation.

For if it be shipped in $(t+1)$ vessels, the moral value of his expectation is

$$
\begin{aligned}
p^{t+1} f(v+s) & +(t+1) p^{t} q f\left(v+\frac{t}{t+1} s\right) \\
& +\frac{(t+1) t}{1 \cdot 2} p^{t-1} q^{2} f\left(v+\frac{t-1}{t+1} s\right)+\cdots+q^{t+1} f(v) .
\end{aligned}
$$

Now $v+\frac{t}{t+1} s=\frac{1}{t+1}(v+s)+\frac{t}{t+1}\left(v+\frac{t-1}{t} s\right)$, $v+\frac{t-1}{t+1} s=\frac{2}{t+1}\left(v+\frac{t-1}{t} s\right)+\frac{t-1}{t+1}\left(v+\frac{t-2}{t} s\right)$, etc. 
Therefore this value is greater than

$$
\begin{aligned}
p^{t+1} f(v & +s)+\left\{p^{t} q f(v+s)+t p^{t} q f\left(v+\frac{t-1}{t} s\right)\right\} \\
& +\left\{t p^{t-1} q^{2} f\left(v+\frac{t-1}{t} s\right)+\frac{t(t-1)}{1 \cdot 2} p^{t-1} q^{2} f\left(v+\frac{t-2}{t} s\right)\right\} \\
& +\cdots+\left\{\left(t p q^{t} f\left(v+\frac{1}{t} s\right)+p q^{t} f(v)\right\}+q^{t+1} f(v)\right. \\
& =p^{t} f(v+s)+t p^{t-1} q f\left(v+\frac{t-1}{t} s\right)+\frac{t(t-1)}{1 \cdot 2} f\left(v+\frac{t-2}{t} s\right) \\
& +\cdots+q^{t} f(v),
\end{aligned}
$$

which is the moral value if it be shipped in vessels.

4. In the above case the merchant may advantageously pay more than the mathematical net premium to insure the cargo.

For suppose he merely pays the net premium $q s$. After paying it his wealth is $(v+p s)$, and its moral value is $f(v+p s)$.

Now taking the general case of $t$ vessels, before insuring, the moral value of his expectation is, as above,

$$
\begin{aligned}
p^{t} f(v+s) & +t p^{t-1} q f\left(v+\frac{t-1}{t} s\right)+\frac{t(t-1)}{1 \cdot 2} p^{t-2} q^{2} f\left(v+\frac{t-2}{t} s\right) \\
& +\cdots+q^{t} f(v) .
\end{aligned}
$$

Applying the corollary, we find this to be less than

$$
\begin{aligned}
f\left\{p^{t}(v\right. & +s)+t p^{t-1} q\left(v+\frac{t-1}{t} s\right)+\frac{t(t-1)}{1 \cdot 2} p^{t-2} q^{2}\left(v+\frac{t-2}{t} s\right) \\
& \left.+\cdots+q^{t} v\right\} \\
& =f\left\{v(p+q)^{t}+p s(p+q)^{t-1}\right\} \\
& =f(v+p s),
\end{aligned}
$$

the moral value after insuring at the net premium. He may therefore without disadvantage pay more than the net premium.

The following diagram illustrates the case where $t=1$.

In this diagram the equation of the curve $P S R Q$ is supposed to be $y=f(x)$. On the axis of $x$ points $M, N, K$ are taken, so that $O M=v ; M N=s$; and $M K=p s$; and therefore

$$
O N=v+s \text { and } O K=v+p s .
$$

At $M, N$, and $K$ ordinates are erected meeting the curve at $P, Q$, and $R$, respectively, so that we have

$$
M P=f(v), N Q=f(v+s) \text {, and } K R=f(v+p s) .
$$


The chord $P Q$ being drawn meets $K R$ at $T$, so that

$$
K T=p f(v+s)+q f(v) .
$$

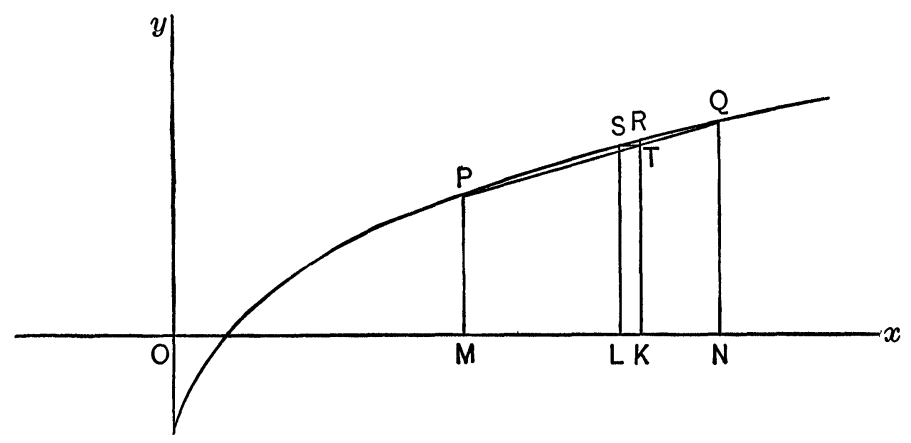

Through $T$ draw $T S$ parallel to the axis of $x$ to meet the curve in $S$, and through $S$ draw the ordinate $S L$ meeting the axis of $x$ in the point $L$; then since $S L=T K$, we have

$O L=$ fortune in possession whose moral value equals the moral value of the expectation.

$L N=$ premium which may, without disadvantage, be paid to insure $S$, represented by $M N$.

$K N=$ net premium for same.

$L K=S T=$ loading allowed.

Ottawa, July 12, 1895.

\section{NOTES.}

A regular meeting of the American Mathematical SociETY was held in New York, Saturday afternoon, October 26, at three o'clock, the President, Dr. Hrus, in the chair. There were nineteen members present. On the recommendation of the Council, the following persons, nominated at the preceding meeting, were elected to membership: Professor Henry Byron Newson, University of Kansas, Lawrence, Kas.; and Professor Frederick Shenstone Woods, Massachusetts Institute of Technology, Boston, Mass. Seven nominations for membership were received. The President announced to the Society that an invitation had been received from the President of the Northwestern University, Evanston, Ill., inviting the Society to hold its next summer meeting at 